abnormalities. Although MRI does identify these lesions, clinical additional value is limited. Improved safety, better availability and tailored procedures are essential for MRI to increase its value in clinical care.

\section{PS-332 BRAIN TISSUE VOLUMES AT TERM-EQUIVALENT AGE IN PRETERM INFANTS: BIOMARKER FOR NEURODEVELOPMENTAL OUTCOME UNTIL 5 YEARS OF AGE}

K Keunen', 'BJM Kooij van, ${ }^{2} \mathrm{P}$ Anbeek, ${ }^{2} \mathrm{I}$ Isgum, ${ }^{1} \mathrm{IC}$ Haastert, ${ }^{1} \mathrm{MA}$ Helvoort, ${ }^{3}$ RAJ Nievelstein, ${ }^{1} \mathrm{~F}$ Groenendaal, ${ }^{2} \mathrm{MA}$ Viergever, ${ }^{1} \mathrm{LS}$ Vries de, ${ }^{1} \mathrm{MJNL}$ Benders, ${ }^{4}$ MJNL Benders. ${ }^{1}$ Neonatology, University Medical Center Utrecht, Utrecht, Netherlands; ${ }^{2}$ Image Sciences Institute, University Medical Center Utrecht, Utrecht, Netherlands; ${ }^{3}$ Radiology, University Medical Center Utrecht, Utrecht, Netherlands; ${ }^{4}$ Centre for the Developing Brain, King's College, London, UK

\subsection{6/archdischild-2014-307384.631}

Objectives To assess the association of brain tissue volumes at term-equivalent age (TEA) with long-term neurodevelopment.

Methods 108 preterm infants (median GA 28.6 weeks; 25.030.9 weeks) were prospectively studied at TEA (median 41.6 weeks PMA; 39.7-43.6). Volumes of eight different tissue types were quantified using an automatic segmentation method (Anbeek, PLOSOne2013)and related to neurodevelopmental outcome using cognitive (CCs), fine motor (FMss), and gross motor scaled scores (GMss) of the BSITD-III at two years corrected age, Griffiths Mental Development Scales (DQ) at age 3.5, and WPPSI at age 5.5. Corrections were made for PMA at scan, intracranial volume and maternal education.

Results Significant results are presented in the table. Both ventricular (Vent) and cortical grey matter volume (CoGM) were inversely related to all included subscales of the BSITD-III and DQ. However, the association at age 3.5 was lost after excluding infants with severe brain lesions (venous infarction, PHVD with neurosurgical intervention, and severe cerebellar haemorrhages). CoGM volume demonstrated a borderline significant inverse correlation with performal IQ at age 5.5 (coefficient-3.2;-6.6-0.08), that did not change after adjustment for severe brain lesions. Cerebellar volume was related to cognitive outcome at 2 and 3.5 years, but the association was mediated by cerebellar injury.

Conclusion Vent and CoGM volumes at TEA may serve as biomarkers for long-term neurodevelopmental outcome in preterm infants. The relationship between larger CoGM volumes and adverse neurodevelopment may reflect disturbances in white matter-CoGM boundaries and warrants further investigation.

\begin{tabular}{lllll}
\multicolumn{6}{l}{ Abstract PS-332 Table 1} \\
\hline & CCs $(n=108)$ & FMss $(n=108)$ & GMss $(n=108)$ & DQ $(n=94)$ \\
\hline Ventricles & $-3.5 ;-6.1-0.9$ & $-0.8 ;-1.3-0.2$ & $-0.5 ;-0.9-0.1$ & $-2.1 ;-3.8-0.4$ \\
Cerebellum & $5.4 ; 1.0-9.7$ & ns & ns & $4.0 ; 1.5-6.5$ \\
CoGM & $-1.6 ;-2.8-0.5$ & $-0.4 ;-0.7-0.2$ & $-0.4 ;-0.5-0.2$ & $-0.9 ;-1.7-0.2$ \\
\hline
\end{tabular}

Numbers reflect coefficients and $95 \%$-confidence intervals

\section{PS-333 DO CLINICAL RISK FACTORS AFFECT WHITE MATTER MICROSTRUCTURAL INTEGRITY (FA) AT TERM EQUIVALENT AGE IN A MULTI-CENTRE COHORT OF PRETERM NEONATES?}

${ }^{1} \mathrm{~J} \mathrm{Schuurmans},{ }^{1} \mathrm{KJ}$ Kersbergen, ${ }^{1} \mathrm{~F}$ Groenendaal, ${ }^{2} \mathrm{~A}$ Leemans, ${ }^{2} \mathrm{MA}$ Viergever, ${ }^{1} \mathrm{~K}$ Keunen, ${ }^{3} \mathrm{~V}$ Fellman, ${ }^{4} \mathrm{AC}$ Berg, ${ }^{1} \mathrm{~F}$ van Bel, ${ }^{1} \mathrm{LS}$ de Vries, ${ }^{5} \mathrm{Mjnl}$ Benders. ${ }^{1}$ Perinatology, Wilhelmina Children's Hospital University Medical Center Utrecht, Utrecht, Netherlands; ${ }^{2}$ Image Sciences Institute, University Medical Center Utrecht, Utrecht, Netherlands; ${ }^{3}$ Pediatrics,
Lund University and Skåne University Hospital Helsinki University, Lund Helsinki, Sweden; ${ }^{4}$ Pediatrics, Lund University and Skåne University Hospital, Lund, Sweden; ${ }^{5}$ Perinatology Centre for the Developing Brain, Wilhelmina Children's Hospital University Medical Center Utrecht King's College London, Utrecht London, Netherlands

\subsection{6/archdischild-2014-307384.632}

Background and aims We aimed to investigate the effect of clinical risk factors on white matter structural integrity at term equivalent age (TEA), as measured by fractional anisotropy (FA). Methods Diffusion tensor imaging of sufficient quality was available for 182 infants (gestational age (GA) $<28$ weeks), scanned at TEA in two centres. FA values of 14 regions (posterior limb of the internal capsule (PLIC), cerebral peduncles, corpus callosum, sagittal stratum, superior/posterior corona radiata, posterior thalamic radiation, left and right side for all) were automatically calculated (ExploreDTI, Leemans ISMRN 2009) using an atlas-based approach (Oishi, NeuroImage 2011). Subjects with overt parenchymal injury were excluded. Clinical characteristics tested against FA in the multivariable linear regression analysis for each region were GA, gender, intra-uterine growth retardation (IUGR), hypotension, mechanical ventilation $>7$ days, morphine (yes/no), post-haemorrhagic ventricular dilatation (PHVD), surgery, postmenstrual age at scanning (PMA) and participating centre. A cut-off value of $p<0.004(0.05 / 14)$ was used to correct for multiple comparison.

Results Statistically significant positive associations with FA were found for PMA in PLIC (right), Superior Corona Radiata and Posterior Corona Radiata (left) and GA in Corpus Callosum, Sagittal Stratum (left), Posterior Thalamic Radiation (left). Statistically significant negative associations with FA were found for Surgery in Sagittal Stratum (right) and IUGR in Posterior Thalamic Radiation (left). Centre was significantly associated with FA in $8 / 14$ brain regions.

Conclusion The microstructure of the preterm brain at TEA depends on GA and PMA at scan, IUGR and surgery. A possible interaction between surgery and morphine warrants further investigation.

\section{PS-334 BIOMARKERS OF DELAYED BRAIN DEVELOPMENT IN A PIG MODEL OF PRETERM BIRTH}

${ }^{1}$ A Bergström, ${ }^{1} \mathrm{~A}$ Rosenørn, ${ }^{1} \mathrm{AD}$ Andersen, ${ }^{1} \mathrm{~T}$ Thymann, ${ }^{1} \mathrm{SS}$ Kaalund, ${ }^{2} \mathrm{E}$ van der Beek, ${ }^{1} \mathrm{P}$ Sangild. 'Clinical and Experimental Nutrition, University of Copenhagen, Frederiksberg C, Denmark; ${ }^{2}$ Early Life Nutrition, Danone Nutricia, Singapore, Singapore

\subsection{6/archdischild-2014-307384.633}

Background and aims Neurodevelopmental seqeulae of premature birth involve cognitive and motor deficits, often persisting into adult life. The molecular mechanisms involved remain yet to be elucidated, but certain regions e.g. cerebellum and neocortex appear particularly sensitive. The current study aimed to evaluate the relevance of Brain derived neurotropic factor (BDNF), involved in the formation of synaptic connexions, and Sonic Hedgehog ( $\mathrm{SHH})$, important for perinatal neuronal differentiation, as potential biomarkers of brain development.

Methods Piglets were born via planned C-section either at full term (gestational age $118 \mathrm{~d}$ ) or 12 days preterm. Euthanization and brain dissection was performed at postnatal day $5(\mathrm{n}=11$, $\mathrm{n}=33)$ and day $26(\mathrm{n}=22, \mathrm{n}=18)$, for terms and preterms respectively. BDNF and $\mathrm{SHH}$ levels were analysed by ELISA in pig cerebellar homogenates. Western blotting (WB) of downstream targets for BDNF (TrkB) and SHH (Patched, Smoothened, Gli-1) were included together with qPCR-array of 84 
neurogenesis pathway related genes (including Bdnf and Shh) on cerebellar and prefrontal cortical tissue.

Results Overall BDNF analysis showed no differences between term and preterm brains but levels were significantly different between day 5 and 26 in preterms only. SHH appeared to be lower in preterms compared to terms, but only significantly on Day 26.

Conclusions The reduced levels of SHH, specifically at day 26, suggest that SSH may be a useful biomarker for delayed brain development and indicate that the pig may provide a relevant model to study the premature brain.

\section{PS-335 PRETERM AND TERM PIGLETS SHOW SIMILAR POSTNATAL ELECTROENCEPHALOGRAPHY (EEG)}

${ }^{1} \mathrm{AM}$ Plomgaard, ${ }^{2} \mathrm{AD}$ Andersen, ${ }^{3} \mathrm{TH}$ Petersen, ${ }^{2} \mathrm{~T}$ Thymann, ${ }^{2} \mathrm{PT}$ Sangild, ${ }^{1} \mathrm{G}$ Greisen. ${ }^{1}$ Department of Neonatology, Copenhagen University Hospital Rigshospitalet, Copenhagen, Denmark; ${ }^{2}$ Department of Nutrition Exercise and Sports Faculty of Science, University of Copenhagen, Frederiksberg, Denmark; ${ }^{3}$ Research Unit on Brain Injury Neurorehabilitation Copenhagen. Department of Neurorehabilitation TBI Unit, University of Copenhagen, Glostrup, Denmark

\subsection{6/archdischild-2014-307384.634}

Background Electroencephalography (EEG) changes rapidly with maturation of the brain in preterm infants. Amplitude-integrated EEG (aEEG) becomes more continuous, bandwidth narrows, and frequencies switch towards faster rhythms, as the child grows older. In preterm infants with brain damage this maturation is typically delayed. We are in the process of developing a preterm piglet brain model. Here we present the results of the EEG as a measurement of brain development in 1-11day-old preterm and term piglets.

Methods One hour of EEG was recorded in 31 preterm piglets aged 1, 2, 4 and 11 days and in 10 term piglets aged 2 and 11 days. All piglets were delivered by C-section at either $90 \%$ or $100 \%$ gestation. Upper and lower margins of the aEEG band were visually identified and bandwidth calculated as the difference between the two values. Spectral analysis of the raw EEG was used to determine the relative power in the delta- $(0.5-3$ $\mathrm{Hz})$, theta-(4-7.5 Hz), alfa- $(8-12.5 \mathrm{~Hz})$, and beta- $(13-30 \mathrm{~Hz})$ bands. General linear models were used with term vs. preterm, and age as predictors.

Results All aEEGs were continuous. The overall means (SD) of upper and lower margin, and bandwidth were $7 \mathrm{mcV}$ (1.7), 15 $\mathrm{mcV}$ (5.7), and $8 \mathrm{mcV}$ (4.3). Upper-, lower margin, bandwidth, alpha, beta and delta bands were unaffected by the predictors whereas the theta band was negatively correlated to age.

Conclusions The preterm piglet EEG was continuous already 10 days prior to term. Thus, maturation of EEG was neither seen in preterm nor term piglets and EEG may not be useful for studying perinatal brain maturation.

\section{PS-336 INFLUENCE OF N-ACETYLCYSTEINE AMIDE (NACA) ON THE INFLAMMASOME PATHWAY. A STUDY ON NEONATAL PIGS}

${ }^{1} \mathrm{~T}$ Benterud, ' $\mathrm{M}$ Ystgard, 'L Pankratov, ${ }^{2} \mathrm{~S}$ Norgren, ${ }^{1} \mathrm{R}$ Solberg, ${ }^{1} \mathrm{OD}$ Saugstad. ${ }^{1}$ Department of Pediatric Research, Oslo University Hospital, Oslo, Norway; ${ }^{2}$ Department of Pediatric Medicine, Karolinska University Hospital, Stockholm, Sweden
Abstract PS-336 Table 1 Study design: Fifty-four newborn piglets, age 12-36h, were included. Invasive blood pressure, EEG and ECG were measured continuously. One control group $(n=6)$ and 4 experimental groups $(n=12)$, exposed to global hypoxia, until BE was either -15 or $-20 \mathrm{mmol} / \mathrm{l}$ (moderate/severe asphyxia with or without NACA) The pigs were observed for $9.5 \mathrm{~h}$

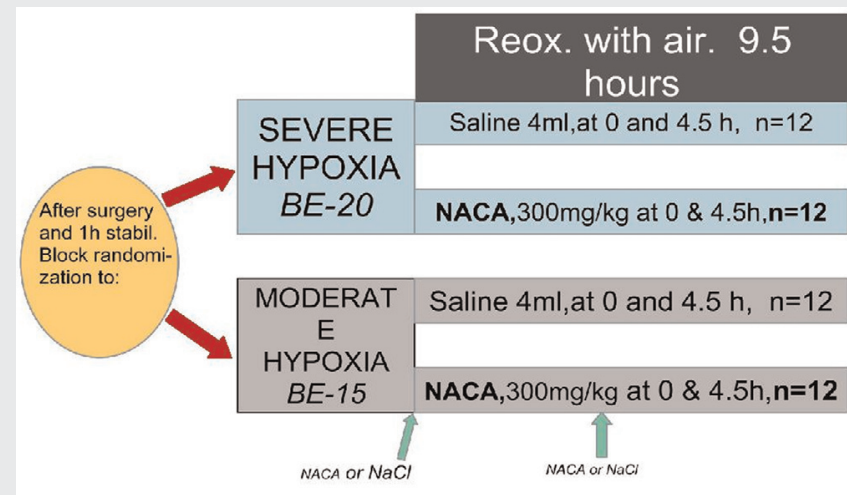

\section{CONTROL $\quad n=6$}

Background and aims Severe perinatal hypoxia contributes to approximately $6 \%$ of spastic cerebral palsy (CP). Studies have indicated an association between elevation of IL-1beta after perinatal asphyxia and the development of CP. The NLRP3 Inflammasome complex may lead to release of the cytokines IL-1beta and IL-18 and cell death. Reactive oxygen species (ROS) have been proposed to be an upstream inducer of this complex and the anti-oxidant N-Acetylcysteine amide (NACA) may provide organ protection after hypoxia.

Objectives To map inflammasome activation in specific brain regions of the pig after neonatal hypoxia-reoxygenation and to investigate if the expression of different proteins in this pathway are modulated by NACA.

Methods

Study design (Table 1).

ELISA was used to measure IL-1b protein in cerebral cortex and Realtime PCR for mRNA expression of NLRP3, ASC, IL-1b and Il18 in cortex, cerebellum, hippocampus and striatum.

Results After severe hypoxia the protein expression of IL-1b in cerebral cortex was reduced for the NACA treated pigs vs. saline, $\mathrm{p}<0.05$.

Abstract PS-336 Table 2 Fold change for NLRP3, ASC, IL-18 and IL-1b measured in cerebral cortex. Significant difference in Fold Change of $\operatorname{ASC}\left({ }^{*}\right), p<0.05$. All values are Median values

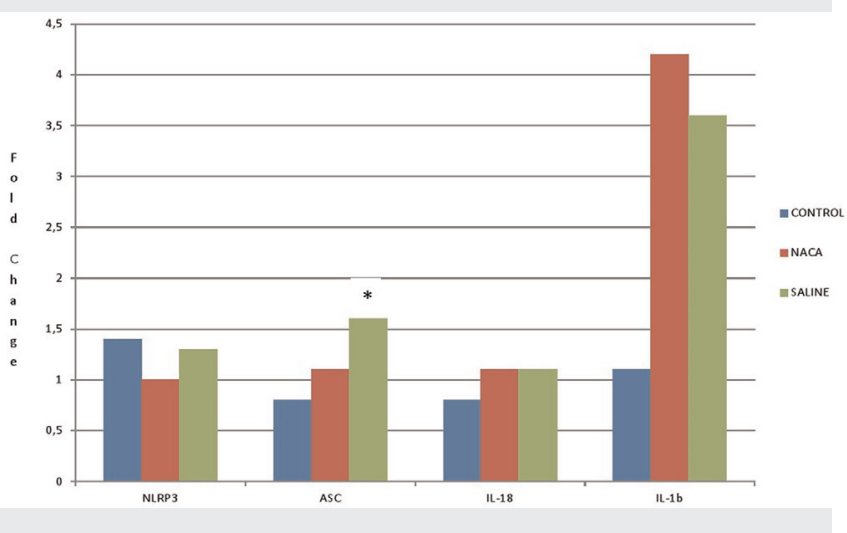

